or other of its noxious effects, may aggravate the symptoms, may tend to produce their return more readily, may make them more difficult of cure; and thus, I think. we can have no difficulty in admitting that the employment of mercury, under such circumstances, may add to the difficulties which attach to the disease itself. I cannot, however, for my own part, see any evidence that mercury is capable of producing those effects which we are in the habit of observ. ing from syphilitic poisons in cases where no mercury is used. Certainly, there are sufficient prejudicial effects arising from its use without adding to the catalogue those which do not belong to it; in fact, all we want to know is, the truth; all we want to know is, what results the remedy is capable of producing, and what it is not; not what evil effects are produced by it, and how few good effects, thereby carrying our notions against it to the extreme; for the remedy is undoubtedly a very valuable and veryiefficacious one; and by leaning to all the prejudices against it we might be led, in consequence of some of the noxious influences that are exerted by it, to neglect its ${ }^{*}$ use altogether. A consideration simply of those prejudicial effects of the remedy, would, of course, naturally lead us to restrict the employment of it in cases where we might otherwise deem it absolutely necessary, and also to endeavour to procure the effect we wish by as small a portion as can be used.

This naturally leads to two questions, first, whether a slight degree of action of the mercury on the mouth, may be consi. dered a proof that it has produced all the effect necessary for the removal of the venereal disease, or whether a more considerable effect is necessary generally, or in particular cases? It has been much the habit in modern times to produce a sensible effect on the moath, and then to discontinue the mercury, under a notion, that when the mouth is offected at all, the system bas experienced a suficient influence for the removal of the disease. I cannot coincide with this opinion. In a great number of instances, a slight effect of mercury on the mouth is sufficient; but there are instances in which that slight effect does not remove the symptoms, and in which, when the remedy is carried further, so as to produce a more con. siderable influence, the symptoms give way. In fact. I think we never see the symptoms of syphilis yield so rapidly, and so favourably, as in certain cases where the remedy, perhaps without our wishing it, has produced a pretty profuse saivation. Under these circumstances, we may notice a sudden and rapid amelioration of the symptoms, which we are not in the habit of see. ing when the mouth is affected in a slighter degree only. Another question immediately connected with the same point, is, how long the remedy should be continued? Is it sul. ficient to destroy the venereal character of a sore, and to produce thel bealthy process of restoration? Would you leave it off, then, and leave the cicatrisation to form of itself? May you discontinue the use of the mercury the moment the cicatrisation is complete, or should you try to secure the patient from the occurrence of secondary symptoms, by proceeding with the employment of the remedy after that? These are important questions, and we have not, perhaps, the means of answering them satisfactorily. With respect to the first, however, it is certainly not safe to discontinue the use of the mercury before the sore is cicatrised all over. Then, se. condly, Is any good produced by continuing the use of the mercury after the cicatrisation is complete, with a view of prerenting a return of the symptoms? This is a very important question, and if you refer to the best writers on the subject, you will find but very little to assist you. Mr. Hunter's observations on this point are very confused and contradictory; sometimes be says, you may discontinue the use of the remedy as soon as the local symptoms have disappear. ed; in other parts, he says, he thinks the further use of mercury will "protect the constitution," to use his own phrase : you will, in fact, get no clear evidence from him. General experience, however, has led to a belief that perseverance in the use of mer. cury for some time, say a week, ten days, or a fortnight after cicatrisation, lias a bene. ficial effect in protecting the constitution; so that persons, when they have used mercury to the extent I have mentioned, are not in the habit of suddenly discontinuing it, but of carrying it on for a short time after the apparent removal of the disease, under a belief that its continuance tends to prevent the recurrence of further evil.

\section{EDINBURGH UNIVERSITY.}

\section{CLINICAL LECTURES IN MEDICINE,}

$\mathrm{BY}$

\section{DR. ALISON. \\ Monday, February lst. \\ CONTAGIOUS FEVER.}

Since the preceding lecture, Dr. Alison said, four new cases had been adnitted, three of which were cases of fever, and no doubt contagious; of these, two were boys, whose symptoms were mild and general, so loeal affection of any importance existing, 
in addition to the ordinary signs of pyrexia, |breathing (previonsly slightly embarrassed) there being only some abdominal pain, became extremely laborious, evidently dewithout tenderness or diarrhca. Several pending on an inflammatory affection of the circumatances tended to prove the contagi- air passages, and he died on the evening of ous nature of these eases; in both, other the tenth day from the beginning of his illmembers of their families were at present ill of fever, and the father of one of the boys, Bowen, senior, showed symptoms of an eminently malignant character. Such were, perhaps, to be expected in a patient of his age (51), habits, and distressed condition; lie had been already confined to his bed six days, and there was evidence of the disease having existed in a premonitory or latent state for some days previous; there were also pneumonic symptoms present. He was restless and delirious, and occasionally showed a disposition to coma and subsultus. His pupils were contracted, his pulse frequent, soft, and feeble, tongue dry and black, bowels confined. These symptoms, especially the condition of the tongue and pulse, Dr. Alison considered gufficient to contra-indicate venesection; he has therefore as yet been treated with laxatives, and calomel, and ipecacuanba. His head has been shaved, and rubbed with oil of turpentine, and he has been allowed a little wine. Under this treatment there bas been some improvement within the last two days, the pulse having become less frequent and more firm; under these circumstances, recovery occasionally takes place. Still the chances are, here, that the circulating forces may fail again, particularly since the tongue continues as dry as ever.

FATAL CASE OF SMALL-POX.

Joln M'Clean, wat. 31 , was admitted on the 28th January, the sixth day after febrile aymptoms had first been observed; the disease was characterised by the eruptive fever, and by the papulse containing watery mat. ter, and having the depression on their tops so peculiar to this affection; it was, besides, marked by the slow progress of the eruption itself; the nature of the eruption was rather confluent tlan distinct, especially on the forehead, and a few indistinct papula were scattered over the internal fauces. The face had swelled as usual; but the swelling of the hauds, which usually follows in favourable cases, had not occurred here. In this man the accompanying fever was rather of the typhoid character; his pulse was soft, tongue dry, and bowels costive: the papulæ, too, were unusually flittened; all which symptoms were indicative of a ferble condition of the circulation, and unfarourable termination of the disease. It was moreorer a case in which no modifuing action could have intluence. The immediate cause of death, however, was of a different nature. On the morning of the s1st, his ness, being one day earlier than small-pox usually proves fatal, and six days after the first appearance of the eruption. Cases of small-pox, attended with such general marks of depression, repeatedly prove fatal, without the occurrence of any local affection, but are more frequently so in consequence of local inflammation, such as occurs in the progress of ordinary fever, and took place in this individual instance. The air passages in particular are liable to these inflammatory affections. In this patient it was seen in an unusual degree of severity ; on the first increase of dyspnoea, leeches were applied, and the hremorrhage they occasioned was so profose, that the application of the sulphate of copper became necessary before it could be restrained ; so abundant was it, that further depletion could not be thought of. Various other auxiliary measures, such as the inhalation of vapour, were also practised, but without the slightest success, as he sunk within twenty-four hours after the aggravation of the local symptoms.

Dr. Alison said, he would reserve further remarks on this case till the post-mortem investigation had taken place. He did not, however, expect to find any very striking effects of the inflammation in the larynx or tracber. Ast to the genesal treatment of small-pox, he observed, that active practice was principally useful during the eruptive fever, which, by the judicious use of cold and purgatives, might be considerably prolonged, and the period of maturation proportionably shortened. The eruption once having appeared, it was useless or hurtful to attempt to interfere with its progress, as it had a certain and necessary course to run. In this stage, the most rational end of practice, was, by the use of gentle laxatives, and the antiphlogistic regimen, to guard against the accession of local symptoms, and should any such supervene, they were to be treated as the circumstances of the case would point out, always bearing in mind the strength of the patient, and the perind of the disease at which they occurred.

Dr. Alison then noticed, at some length, a case of hydrocephalus, successfully trented by repeated leeching and bleedings, and proceeded to recapitulate the leading features of the remarkable cases which occurred during his decision of the clinical course; his observations, however, were no more than condensed views of the reports we have already given; he requested the class to meet him ayain on the ensuing Wednesday, when be hoped to communicate to them the 
account of the dissections of the recent fatal cases of small-pox and diptherite. On the day named, however, Dr. Alison stated, that permission had been refused to examine the bodies of these patients. The circumstance was unusual, and peculiarly unfortunate, as these cases were, perhaps, the most interesting which have occurred during tho course.

\section{Wednesday, February $4 t h$. DIPTHERITE.}

Dr. Alison delivered his concluding lecture. He first referred to the case of Millar. The leading facts that should be remembered in this case, Dr. Alison said, were, the inflammation of the left tonsil, soft palate, and uvula, as it appeared on the day of his admission (which was the fourth of the disease), characterised by the diffused swelling, pain, deep-red colour, and the effusion of coagulable lymph, which last appearance was by no means more abundant than usually happens in slight inflammatory affections of these parts; his illness was attributed to cold, and the accompanying fever was mild (the pulse being 96), though of decidedly inflammatory type. Under these circumstances, the ordinary termination to be expected was, suppuration of the tonsil, under which impression, and on account of the mildness of the fever, local measures were preferred to any general depletion; and the tonsil was scarified, and leeches externally applied. No discharge of pus, however, followed the scarification; but there was a copious flow of blood, and the leeches also operated well. During this time, the difficulty of deglutition was slight, nor was the breathing much affected; but on the eighth day, the tendency of the infiammation to spread down the air passages was first apparent, and continued to increase, despite every measure attempted for the patient's relief. Dr. Alison reminded the class, that after this tendency to spread bad shown itself, two copious bleedings iad been practised without benefit ; leeches bad been repeatedly applied; emetics, puryatives, calomel, and nauseating remedies, ilso tried without success : locally, the nitrate of silver was used, and alum in powder blown upon the inflamed parts; yet the case proved fatal within thirty-six hours after the extension of the inflammation had taken place.

Cases of this description, he continued, were fortunately rare in adults, yet had been repeatedly mentioned by authors, and two varieties of the disease had been described, the distinction between which was of much importance, as a preternatural membrane was formed in one and not in the other; one had been termed the cynanche laryngia, and was well described by Dr. Farre; the latter named the true croup. (A preparation was here sent round of croup in a clild, attended with the formation of the false membrane, which extended down the trachea nearly to the bifurcation). The peculiarity of (Ii]lar's case was, that it did not strictly belong to either of these divisions; in him false membranes had manifestly been formed, $y+t$ the disease set in with symptoms of actue inflammation, which in the true croup of adults was an anusual occurrence ; in adults, whenever the tonsils, uvula, and soft palate, became inflamed, and the accompanying fever was at the same time strictly phlogistic, the local disease rarely extended itself beyond the superior aperture of the trachea, and the chief danger was caused by the thickening or œdema of the glottis, or effusion of pus behind that opening. In such cases, the difficulty of breathing is generally urgent from the beginning, and relief is with more facility obtained, by the general abstraction of blood; the chance, too, of success from the operations of laryngotomy or tracheotomy, is, for many and obvious reasons, greater. According to Dr. Bailie, in these cases the operation should not be deferred longer than thirty hours after the commencement of the disease; and in one example which occurred a few months ago in Dr. Gregory's ward, the patient recorered, tracheotomy having been performed in twenty-four hours after the dyspnoa became severe. Among adults, true croup was generally observed in persons already enfeebled by various causes, such as recent fever, or in the convalescence from inflammatory diseases, the disease commencing in the tonsils, with a trifling degree of swelling, and with typhoid fever, and hence extending down the larynx. In the Arclives de Medicine, there is a paper by Louis, who relates eight cases of croup in adults, seven of which were fatal; in six of which false membranes were found on dissection; in the majority of these examples, the croup had supervened during the convalescence from fever, and in all, the difficulty of deglutition was comparatively slight. A fatal cose, similar to these, occurred to himself (Dr. Alison) not long since, in which the bad symptoms came on in a remarkably insidi. ous form, and in forty-eight hours after its commencement. Antipblogistic measures were evidently no longer suitable; the operation was then proposed, and performed by Dr. Ballingal, between the thyroid and cricoid cartilages. The patient soon sunk; and, on examination, it was found that a new membrane had extended into the trachea, below the artificial opening; lad the incision been made lower down, it is probable the chances of success would have been greater; this was prevented by 